\title{
A catalogue of oxygen-rich pulsating giants in the Galactic halo and the Sagittarius stream ${ }^{\star}$
}

\author{
N. Mauron ${ }^{1}$, L. P. A. Maurin ${ }^{2}$, and T. R. Kendall ${ }^{3}$ \\ ${ }^{1}$ Université de Montpellier, Laboratoire Univers et Particules de Montpellier CNRS/UM, Place Bataillon, 34095 Montpellier, France \\ e-mail: nicolas .mauron@umontpellier.fr \\ 2 Observatoire des Ifs, 6 impasse des Ifs, 84000 Avignon, France \\ 318 Market Square, Northampton NN1 2DL, UK
}

Received 14 August 2018 / Accepted 27 March 2019

\begin{abstract}
To construct a catalogue of oxygen-rich (M) asymptotic giant branch (AGB) stars in the halo, complementing the catalogues of carbon-rich (C) stars, previous lists of Miras and SRa semi-regulars located in the northern hemisphere are merged and cleaned of various defects. After putting aside known $\mathrm{C}$ stars, characteristics such as colours and periods indicate that most of the remaining objects are M stars. Distances are obtained through the period-luminosity relation. By considering their position in the sky, stars lying at $|Z|>5 \mathrm{kpc}$ are confirmed to be in majority in the Sgr tidal arms. The M stars are more numerous than C ones. Our distance scale is supported by two cool variables located in the Pal 4 globular cluster. Along the Sgr arms, there is reasonable agreement on distances of our objects with recent RR Lyrae distances. A few stars may be as distant as $150 \mathrm{kpc}$, with possibly four at the trailing arm apocentre, and two in the A16 sub-structure, angularly close to two C stars. Ninety radial velocities are collected from Gaia and other sources. A catalogue with $417 \mathrm{M}$ pulsating AGB stars is provided. This catalogue contains $\sim 260$ stars in the halo with $|Z|>5 \mathrm{kpc}$. Their $K_{s}$ magnitudes range from 8 up to 13 . For comparison, the catalogue also provides $\sim 150$ stars in the disc having $5<K_{s}<8$.
\end{abstract}

Key words. Galaxy: halo - Galaxy: stellar content - stars: AGB and post-AGB

\section{Introduction}

Stars evolving on the asymptotic giant branch (AGB) can be either oxygen-rich $(\mathrm{M})$, or carbon-rich $(\mathrm{C})$. The general properties of AGB stars are reasonably understood and summarised in the book of Habing \& Olofsson (2004). Much previous research concerning Mira variables enabled a greatly improved knowledge of the main components of the Galaxy (bulge, disc, bar, etc). For example, by considering Mira stars close to the Sun, their locations and their kinematics, Feast \& Whitelock (2000) could detect the extension of the Galactic bulge bar in the solar vicinity and beyond. In a galaxy, the presence of AGB C stars is generally interpreted as indicating that an intermediate-age population exists. In contrast, the M AGB stars may be much older since one finds them in globular clusters. The goal of this work is to achieve a catalogue of the M AGB population of the Galactic halo, and we restrict this research to the Mira-type and SRa-type variable stars. In this paper, we name these periodic stars MSRa stars. Also, although this paper is devoted to M stars, we make a detailed comparison with $\mathrm{C}$ stars at several points to highlight differences and similarities.

In comparison with the Galactic disc, the understanding of AGB stars located in the halo has to be improved. A great deal of research has been carried out on $\mathrm{C}$ stars because their very red colour, or their peculiar spectra makes it easier to discover them; see Huxor \& Grebel (2015; hereafter HG2015) for a synthesis. However, in comparison to $\mathrm{C}$ stars, relatively little attention has been devoted specifically to finding $M$ stars. The fact that they

\footnotetext{
* Full Table 3 is only available at the CDS via anonymous ftp to cdsarc.u-strasbg. fr (130.79.128.5) or via http://cdsarc. u-strasbg.fr/viz-bin/qcat?J/A+A/626/A112
}

can be found in globular clusters suggests that there may be such a population in the halo, but, to our knowledge, a systematic census is missing.

One of the difficulties in finding M AGB stars at high Galactic latitude is to separate them from the large amount of dwarfs in the disc. Infrared properties and/or variability may be thought as useful methods. One of the early studies of these M stars was based on the availability of the infrared IRAS data. Indeed, a complete survey of M Miras with short or intermediate period and with $|b|>30^{\circ}$ is due to Jura \& Kleinman (1992), but distances to the Galactic plane $(Z)$ are mostly less than $3 \mathrm{kpc}$. More precisely, of a total of 318 objects, only 13 have $|Z|>3 \mathrm{kpc}$. Another interesting work is that of Whitelock et al. (1994, 1995). To investigate cases of very high-mass loss AGB stars, they choose them in the south Galactic Cap at $b<-30^{\circ}$ and select those that have IRAS fluxes and colours. Because fluxes at $12 \mu \mathrm{m}$ and $25 \mu \mathrm{m}$ are needed, these sources are not very distant: their time-averaged magnitude is typically $K<7$, with $|Z|<4 \mathrm{kpc}$. Finally, Gigoyan \& Mickaelian (2012) published a list of M stars (dwarfs or giants or AGB stars) found by eye-scanning the Byurakan objective-prism plates. We have cross-matched their sample with the General Catalogue of Variable Stars (Samus et al. 2017) and 85 Miras are identified. However, their $K_{s}$ magnitudes are less than seven because the Byurakan plates are not deep.

This situation has changed with large-scale experiments searching for variable objects over all the sky. A first catalogue of periodic giants based on the LINEAR experiment was published by Palaversa et al. (2013). It covers 10000 square degrees and reaches $r_{\mathrm{SDSS}}=18$. A second catalogue is by Drake et al. (2009, 2014), from the Catalina experiment, covering over 20000 square degrees down to $V \sim 19$. In addition to yielding a number 
of new results on variable objects in general, these databases dramatically increase the number of known MSRa out of the Milky Way plane. From their Catalina sample of 500 MSRa, Drake et al. (2014) show that some of these trace the Sgr stream. We decided to reanalyse their data for several reasons. First, we intend to build a catalogue of $\mathrm{M}$ stars in the halo, as a complement to the HG2015 compilation of C stars. Second, in order to determine distances and since periods are provided, we use the $K$-band period-luminosity relation instead of an absolute magnitude $M_{v} \sim-3$ adopted by Drake et al. (2014). Third, the Sgr arms have been mapped recently in detail with RR Lyr variables from the PanSTARRS data, providing accurate distances to which those of MSRa stars can be compared (Sesar et al. 2017; Hernitschek et al. 2017). Finally, we wish to investigate the possible existence of very distant stars, at more than $\sim 70 \mathrm{kpc}$ from the Sun.

In Sect. 2, we construct a preliminary list of MSRa stars from catalogues mentioned above, pay attention to a variety of flaws and obtain a sample of 417 stars. In Sect. 3, we focus on their properties, in particular those independant of the distances: we have examined their colours, (peak-to-peak) $V$-band amplitudes, periods, apparent positions and how to best attribute a pulsation mode through consideration of photometric colours. In Sect. 4, we describe and discuss the results. We also derive distances and their uncertainties. It is found that the number of $M$ stars in the halo from our sample is richer by a factor of $\approx 3$ than the presently known sample of halo $\mathrm{C}$ stars. A few remarkable stars are discussed. Our catalogue of $\mathbf{M}$ stars is presented, and includes 90 radial velocities. Finally, we give our conclusions in Sect. 5.

\section{Building the sample}

To build a preliminary list of MSRas, first we considered the catalogue of Drake et al. (2014). We note that this catalogue is based on the Data Release 1 (DR1) of Catalina, and that supplementary monitoring data are provided in the DR2, which is usable on the Catalina web site. We added the MSRa stars contained in the catalogue of Palaversa et al. (2013). We also added the C stars, periodic or irregular, compiled by HG2015, as well as other $\mathrm{C}$ stars that are warmer, but may have been missed as pulsators in previous studies. Finally, we examined stars that had been found as contaminants in our survey for $\mathrm{C}$ stars. After checking their light curves on the Catalina DR2 database, those which were periodic were included.

Because the LINEAR or Catalina coordinates have an accuracy of $\sim 3^{\prime \prime}$, we cross-correlated our list with the 2MASS catalogue with a search radius of $5^{\prime \prime}$. Objects lacking a 2 MASS counterpart were rejected. In rare cases, it may be that more than one 2MASS source is found. Generally, there is one bright source and the others are much fainter. In such cases, consideration of magnitudes allows us to identify the true counterpart. However, when the choice is not obvious, the object was dropped.

This first 2MASS cross-matching procedure leads to a list of 894 entries. Further cleaning is necessary for many reasons. Numerous objects are common to Drake's and Palaversa's catalogues, and there are 112 duplicates in our preliminary list. These duplicates are, however, interesting as they can inform us about differences in amplitudes and periods provided by the two databases. There are also objects presented in the LINEAR or Catalina catalogues as being periodic variables, but a systematic examination of Catalina light curves shows them to be of poor quality, almost constant, or not clearly periodic. These
Table 1. False very-faint variables.

\begin{tabular}{rrrrrrr}
\hline \hline RA & Dec & $K_{s}$ & $P 1$ & Mira & $P 2$ & $\rho$ \\
\hline 57.8024 & 33.0446 & 16.62 & 419 & RX Per & 422 & 0.95 \\
58.2531 & 25.5515 & 13.87 & 225 & SX Tau & 225 & 0.86 \\
70.5752 & 6.8905 & 15.60 & 413 & BZ Tau & 400 & 1.11 \\
119.2810 & 21.3302 & 14.77 & 338 & XY Gem & 340 & 0.78 \\
257.7185 & 27.0710 & 13.65 & 299 & RT Her & 298 & 1.02 \\
259.7585 & 8.5025 & 14.39 & 266 & V477 Oph & 267 & 0.67 \\
317.8590 & 13.3561 & 15.75 & 277 & AN Peg & 280 & 1.25 \\
326.2447 & 12.7085 & 11.91 & 314 & TU Peg & 321 & 1.54 \\
333.0724 & 14.5785 & 14.72 & 400 & RS Peg & 415 & 1.53 \\
\hline
\end{tabular}

Notes. Their (J2000) coordinates are in given degrees. $K_{s}$ is the faint star magnitude from 2MASS. $P_{1}$ (in days) is its period from Catalina DR2. The polluting star name is indicated. $P_{2}$ is its period from the General Catalogue of Variable Stars. $\rho$ is the angular separation in arcminutes.

objects were rejected, as well as those that are saturated. We also rejected objects with $K_{s}<5$ in the 2MASS catalogue, because they are too close to us, but we keep many disc stars with typically $K_{s} \sim 5-7$, in order to be able to compare halo and disc properties.

Occasionally, a periodic light curve displays a significant amplitude variation $(\sim 30 \%)$. Although this may happen for AGB stars (see below), another possibility is that the star is a still unknown, slowly rotating, spotted giant. In the Galactic bulge, the overwhelming majority of these variables have periods less than 100 days (Drake 2006). To our knowledge, no similar information exists for the thick disc or halo giant populations. This limit of 100 days is smaller than the periods of most of the stars studied in this work. In these occasional cases we chose not to be too conservative at this stage, and we kept these rare cases in our list. An additional reason for doing so is that supplementary observations could resolve this ambiguity. For example, spectra of red, variable members of $47 \mathrm{Tuc}([\mathrm{Fe} / \mathrm{H}] \sim-1.5)$ display strong, variable Balmer emission lines that are explained with pulsation shocks (van Loon et al. 2007; their Fig. 21).

We also interrogated Simbad at CDS and found that two quasars are present in our list: one is at $\alpha=26.13977$ $\delta=27.08419$, and the other at $\alpha=116.99665, \delta=20.87373$, coordinates J2000 in degrees. As a consequence, we systematically looked for matches in the QSO-AGN catalogue of Véron-Cetty \& Véron (2010; 168940 entries), and the Sloan DR7 quasar catalogue (Schneider et al. 2010; 105783 entries), but no supplementary cases were found.

Finally, a more intricate cleaning necessity appeared when we focused our attention on very distant stars, possibly located at more than $\sim 100 \mathrm{kpc}$. We discovered that many of these faint sources $\left(K_{s} \sim 12-14\right)$ with apparent periodic signals were, in fact, objects polluted by bright Miras located at less than $2^{\prime}$ away and with identical periods. A thorough search within $5^{\prime}$ for all our sources resulted into identifying nine cases, given in Table 1.

To conclude, having achieved this cleaning procedure, 417 objects remained that are not known as being carbon rich. We refer to these 417 objects hereafter as our sample.

\section{Analysis}

Since this paper deals with the halo MSRa population, including the Sgr arms, and because we do not have a complete kinematic information for the majority of sources in our sample, we 

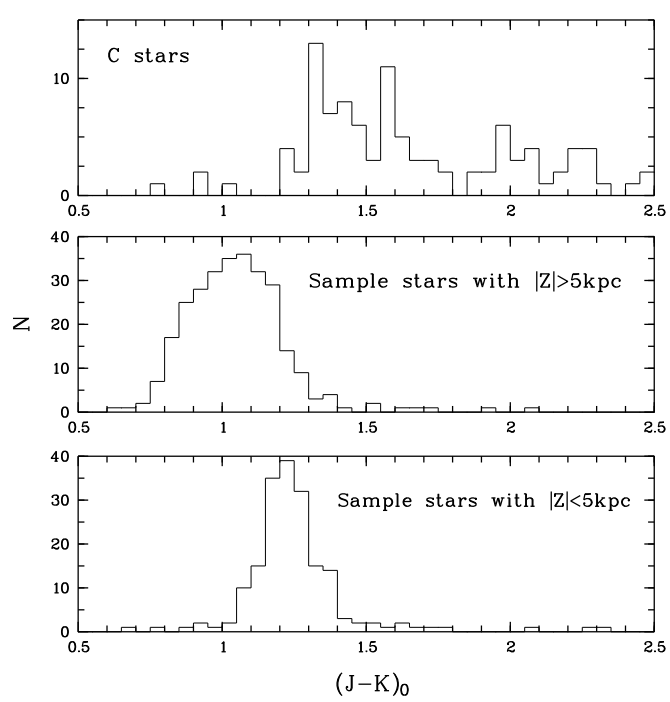

Fig. 1. Histogram of $(J-K)_{0}$ of long-period variables. Top panel: halo C stars. Middle panel: stars in our sample for which the height $|Z|$ from the Galactic plane is larger than $5 \mathrm{kpc}$. Lower panel: as middle panel, but $|Z|<5 \mathrm{kpc}$. We do not include irregular variables, with no well-defined periodicity (they are ignored in this paper) in any of the panels.

needed to consider a quantitative limit separating halo stars and those of the Sgr arms on one side, and a complementary group of objects from the disc, on the other side. After some tries, we found that a separation with the height $Z$ (in kpc) from the Galactic plane works reasonably well, and that $|Z|=5 \mathrm{kpc}$ is a good compromise. The quantity $Z$ is derived by assuming all objects to obey the $K_{s}$-band period-luminosity relation with Wesenheit indices of Soszyński et al. (2007). A more detailed discussion of the determination of distances is done in Sect. 4.1. Taking $Z$ smaller than $5 \mathrm{kpc}$ (i.e. $2-3 \mathrm{kpc}$ ) retains too many disc sources which pollute the halo sub-sample. Adopting a height of $\sim 10 \mathrm{kpc}$ is too large because, at least from the model of Law \& Majewski (2010), the Sgr arms distance can be as close as $10 \mathrm{kpc}$ from the Sun (see also Fig. 15 of HG2015). In the following, we present results concerning the colours, amplitudes, periods, and location in the sky. These are the best-determined characteristics because they do not involve distances.

\subsection{Colours, amplitude and periods}

In Fig. 1, we show three histograms of the $\left(J-K_{s}\right)_{0}$ colour index. Correction for interstellar extinction is achieved with $A_{J}=0.87 E(B-V)$ and $A_{K s}=0.35 E(B-V)$, from Cardelli et al. (1989), with $E(B-V)$ from Schlegel et al. (1998). The upper panel displays $C$ stars. Stars of our sample with $|Z|>5 \mathrm{kpc}$ and $|Z|<5 \mathrm{kpc}$ are shown in the middle and bottom panels, respectively. It can be seen that $\mathrm{C}$ stars are in very large majority redder than $\left(J-K_{s}\right)_{0} \approx 1.3$, while an overwhelming proportion of our stars at $|Z|>5 \mathrm{kpc}$ are bluer. This strongly suggests that these bluer stars are mostly oxygen-rich. The histogram ordinates are the number of objects per bin, and shows that the bluer sample is considerably richer than the $\mathrm{C}$ star sample, by a factor of $\approx 3$. The $\left(J-K_{s}\right)_{0}$ colours in the lowest panel suggest that the stars of our sample with $|Z|<5 \mathrm{kpc}$ are in majority M stars, although a few $\mathrm{C}$ stars may be present.

Pulsation amplitudes deserve attention because they are involved in the uncertainty on distances. This is because distance determination is based on the use of $K_{s}$, but the phase of this $K_{s}$ measurement is ignored. Therefore we attempted to estimate

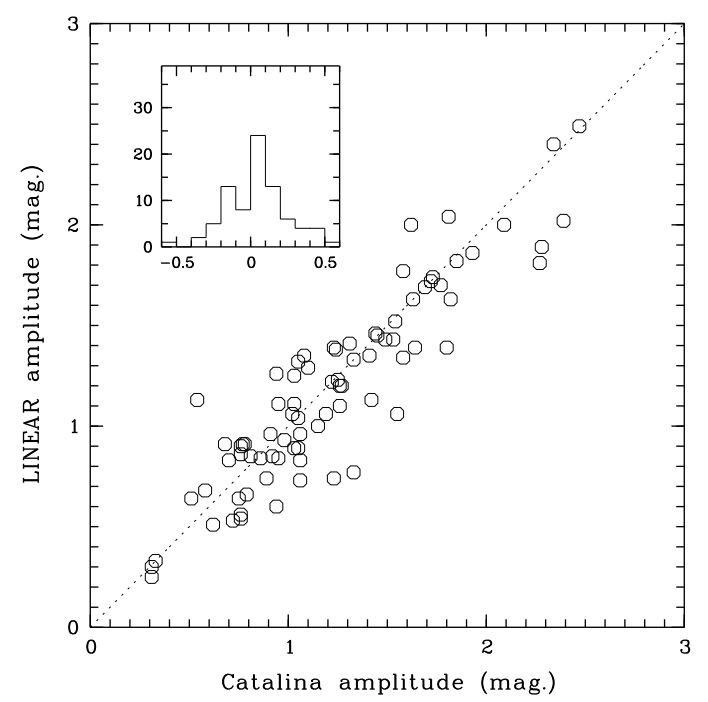

Fig. 2. Catalina versus LINEAR $V$-band amplitudes for common MSRa variables. The dotted line is a 1:1 line. The inset shows the histogram of residuals. The $1 \sigma$ scatter is $0.21 \mathrm{mag}$.

the range of variation in $K_{S}$ by scaling optical amplitudes to the near-infrared. The LINEAR survey took place between 1998 and 2009, while the Catalina survey DR1 covers the period 2005-2011. Thus, an overlap of four years exists. Consequently, we expect that, in a first approximation, amplitudes given by the two experiments should agree. We note that Catalina amplitudes are derived from Fourier fits, while LINEAR amplitudes are the range of values between $5 \%$ and $95 \%$ in the signal distribution. Figure 2 displays one amplitude versus the other for common objects of our initial list. The agreement seems very reasonable, with $\sigma=0.21 \mathrm{mag}$. This scatter means that some amplitudes may change over years by at least this amount.

In Fig. 1, we saw that the halo sample stars (with $|Z|>$ $5 \mathrm{kpc}$ ) are generally bluer than the halo $\mathrm{C}$ stars, although there are exceptions. Here, we have investigated whether a periodamplitude diagram would bring some complementary information. Figure 3, panel a, shows that these halo stars comprise a main population with $P=150-175$ days, and a small population with low amplitude and longer periods. In contrast, panel $\mathrm{b}$ shows that the periods of $\mathrm{C}$ stars are more scattered over a 150-300 days interval, and do not include the large-period low-amplitude family. Therefore, a periodic AGB halo star with period $P<170$ days is more probably of $\mathrm{M}$ type than of $\mathrm{C}$ type. This result is supported by an ongoing program of $\mathrm{M} / \mathrm{C}$ classification of several hundreds of Catalina MSRa in both hemispheres (Gigoyan, priv. comm.). This program shows that, at least for $V \leq 16$, only $\sim 3 \%$ are newly discovered $\mathrm{C}$ stars.

\subsection{Apparent positions}

In Fig. 4 (middle panel), we plot $M$ stars verifying $|b|>20^{\circ}$, and $|Z|>5 \mathrm{kpc}$. With these selections, the disc members nearly vanish. Clearly, a very large number of these stars are located close to the sinusoid fitted on $\mathrm{C}$ stars. There are many more objects with right ascension (RA) between $0^{\circ}$ and $80^{\circ}$, but the most numerous population is in the Sgr leading arm with RA between $160^{\circ}$ and $250^{\circ}$.

\subsection{Separating $C$ and $C^{\prime}$ pulsating sequences}

The MACHO and OGLE surveys (Alcock et al. 1997; Udalski et al. 1994) monitoring stars in the Large Magellanic 

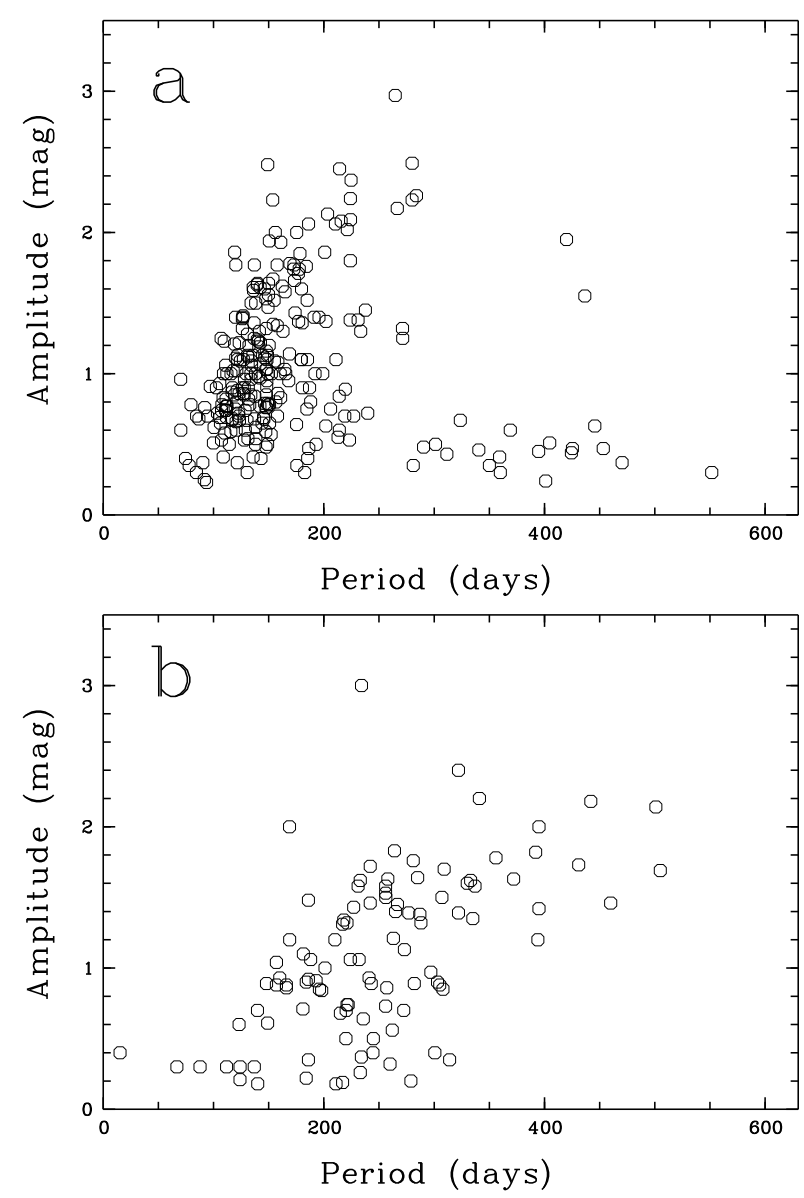

Fig. 3. Catalina amplitudes plotted as a function of pulsation periods for MSRa in the halo. All stars plotted here have $|Z|>5 \mathrm{kpc}$. Panel a: our sample stars obeying $\left(J-K_{s}\right)_{0}<1.3$. Panel $b$ : carbon stars. We note the large difference of these two distributions, strongly suggesting that most of the stars in the upper panel are not carbon rich. All stars plotted here have $|Z|>5 \mathrm{kpc}$.

Cloud (LMC) or the Galactic bulge have shown that MSRa stars pulsate in different modes. Several period-luminosity sequences exist, in particular the so-called sequence $\mathrm{C}$ for fundamental pulsation and sequence $C^{\prime}$ for first overtone (Wood et al. 1999; Ita et al. 2004). Here, in order to avoid confusion with $C$ (carbon) stars, we name seq-C, seq- $\mathrm{C}^{\prime}$, and seq-D the three P-L sequences considered in this work. In the Magellanic Clouds or the bulge, the sequence separation was made possible because all stars were at the same distance. In the case of stars in the halo or in the Sgr arms, this distance is unknown and has to be determined. For carbon stars, HG2015 based their classification seq-C or seq- $\mathrm{C}^{\prime}$ on three diagrams involving the $\left(J-K_{s}\right)_{0}$ colour index, periods and amplitudes. Although, as they note, the diagram $\log (P)$ versus $\left(J-K_{s}\right)_{0}$ presents some difficulties to determine this separation, they reach the conclusion that very few (5 of 121) are seq- $C^{\prime}$ stars.

Concerning our sample stars, none of which are known $\mathrm{C}$ stars, we find that the clearest results are obtained by considering the $\left(I-K_{S}\right)_{0}$ colour. We find this colour to be more efficient than $\left(V-K_{S}\right)_{0}$ to separate sequences equally well for the LMC and the halo sample. Figure 5 shows the situation of LMC stars, for which sequences are known. Here, $I$ is a time-averaged magnitude from OGLE and $K_{s}$ is from the single epoch 2MASS survey. We draw a boundary between seq-C and seq- $\mathrm{C}^{\prime}$ populations. Figures 6 and 7 show the same diagrams for the stars of our sample, with $|Z|>5 \mathrm{kpc}$ and $|Z|<5 \mathrm{kpc}$, respectively. The $I$-band photometry that we use is derived from the Catalina $V_{\mathrm{CSS}}$ magnitude and the 2MASS $J-K_{s}$ colour, as explained in the Appendix.

In Figs. 6 and 7, the plotted boundary lines are identical to those of the LMC. One could object that boundaries separating sequences might change with population characteristics, such as metallicity. Here, we have assumed that there is no change of these boundaries. The metallicity of many giant stars in the Sgr arms is close to that of the $\mathrm{LMC}$, where $[\mathrm{Fe} / \mathrm{H}] \approx-0.5$ on average. More precisely, observations of the metallicity distribution in the Sgr arms can be found in Monaco et al. (2007), Chou et al. (2007), and Carlin et al. (2018), among others. The distribution peaks at $\sim-0.75$ and has a lower metallicity component. Therefore, it seemed reasonable to us to keep boundaries unchanged, although we recognise that other factors, such as star formation history, could play a role. Certainly, our assumption deserves additional investigation.

In Fig. 6, most of our stars are to the right of the boundary. Six objects are clearly to the left, and we assign them to sequence $\mathrm{C}^{\prime}$. The position of about 20 objects, to the right of a dotted line, suggests that they are not members of sequence C. Comparing Figs. 6 and 7 shows that the main cloud shifts to the red and longer periods in Fig. 7. Since disc stars dominate the $|Z|<5 \mathrm{kpc}$ population, one interpretation of this shift is that it arises from the higher metallicity of the disc as compared to the halo. In Fig. 7, we consider that only five stars are separated enough from the boundary line to be considered as being on the sequence $C^{\prime}$. Three stars may lie on the D sequence.

The fact that so few stars are found in sequence $C^{\prime}$ is puzzling, in strong contrast with what is seen in the LMC. We first note that exactly the same fact occurs for halo carbon stars, as found by HG2015. Additionally, the HG2015 method of separating sequences and our method are different, leading however to similar conclusions. HG2015 suggested that seq- $\mathrm{C}^{\prime}$ pulsators have amplitudes too small to be included in the Catalina MSRa catalogue of Drake et al. (2014). This may be true, although not entirely convincing because $\sim 15 \%$ of a total of $1200 \mathrm{LMC}$ seq- $C^{\prime}$ stars have a $V$-band amplitude larger than 0.55 mag in the OGLE catalogue. Such a large amplitude is relatively well detected by Catalina, a point that remains unexplained for us.

\section{Results}

\subsection{Distances}

After having determined the pulsation sequences for stars of our sample, we can derive their distances. For that purpose, we used, as HG2015 did for C stars, the near infrared Wesenheit magnitudes $W_{J K}$. We used the $P-W_{J K}$ relations given in Table 1 of Soszyński et al. (2007) for O-rich sequences labelled $\mathrm{C}_{\mathrm{O}}, \mathrm{C}_{\mathrm{O}}^{\prime}$ and $\mathrm{D}_{\mathrm{O}}$. We adopted $m-M=18.49$ for the LMC distance (e.g. De Grijs et al. 2014).

The uncertainty on distances is comprised of: (a) the measurement error of the $K_{S}$ 2MASS magnitude provided in the 2MASS catalogue, of order $0.03 \mathrm{mag}$; (b) the relative uncertainty on the period found to be $\sim 2 \%$, from comparing periods given by LINEAR and those of Catalina, implying 0.04 mag on the absolute magnitude; (c) the scatter of the P-L relation, i.e. $\sigma=0.15 \mathrm{mag}$; and finally (d) the uncertainty that originates in the single epoch character of $K_{s}$. Ideally we should use a timeaveraged $K_{s}$, but this quantity is lacking. This implies an uncertainty in relation with the $K_{s}$-band amplitude that we estimate as explained below. 

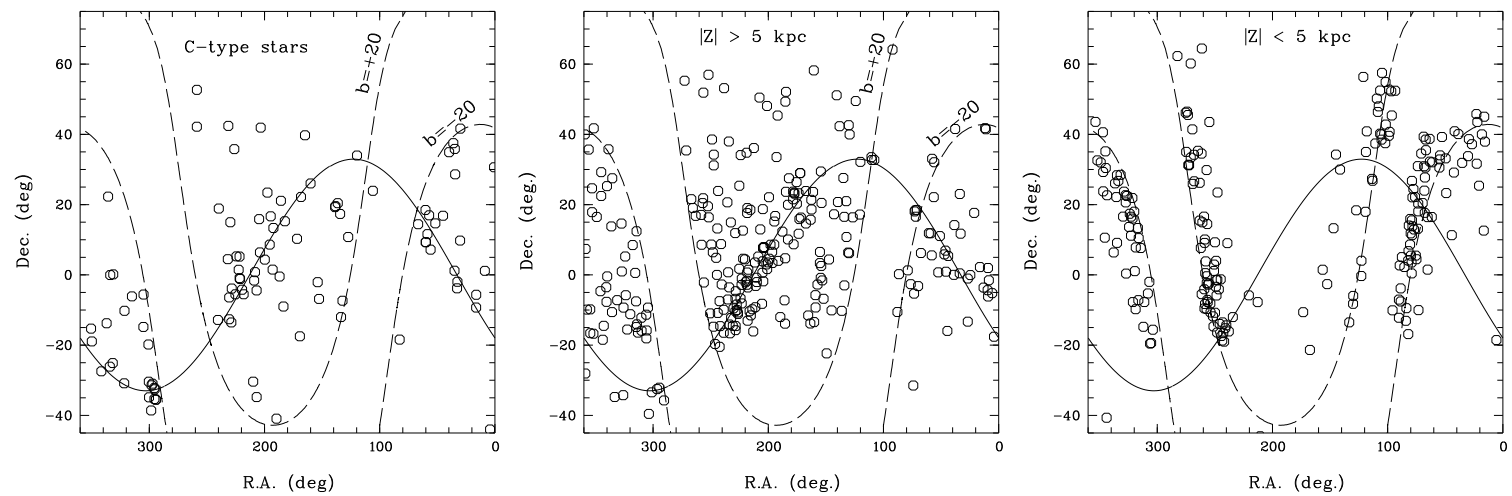

Fig. 4. Apparent positions of pulsating AGB stars in equatorial coordinates. Left panel: all known C stars that are periodic and have $|Z|>5 \mathrm{kpc}$. The sinusoid is an eye fit to the majority of these $\mathrm{C}$ stars. Middle panel: stars of our sample (M type) having $|Z|>5 \mathrm{kpc}$. Right panel: stars of our sample having $|Z|<5 \mathrm{kpc}$. Dashed lines indicate $|b|=20^{\circ}$. We note that $\mathrm{M}$ stars are more numerous than $\mathrm{C}$ stars along the Sgr arms.

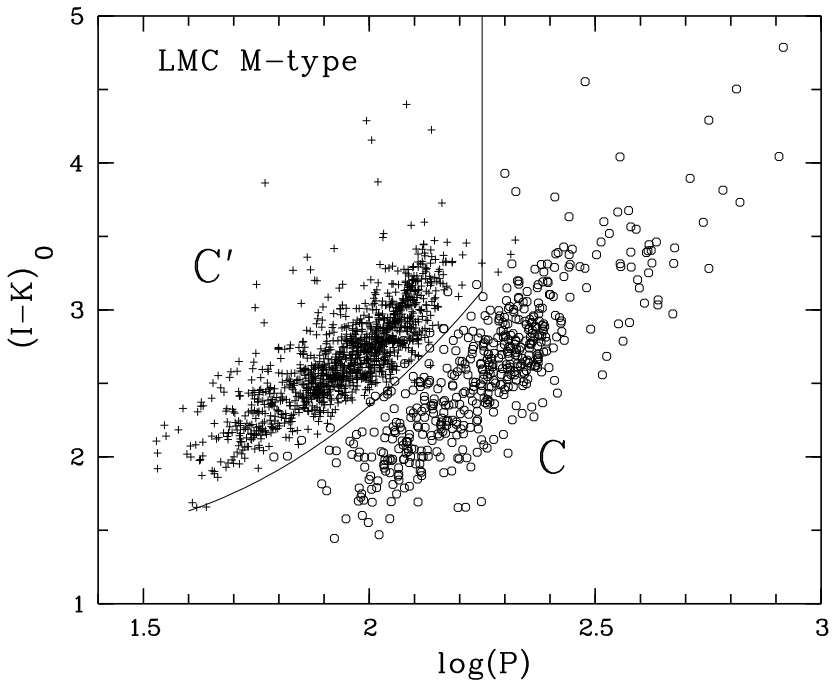

Fig. 5. $\left(I-K_{s}\right)_{0}$ colour index versus period (in days) for oxygen-rich MSRa variables of the LMC. The curved line separating sequence $\mathrm{C}$ and $\mathrm{C}^{\prime}$ is $y=a x^{2}+b$ where $x=\log (P)-1.2, a=2.3, b=1.45$. The vertical line corresponds to $P=178$ days.

For C stars, HG2015 exploit a correlation between the $K_{s}$ amplitude and the $\left(J-K_{s}\right)_{0}$ colour, established for dusty Miras with that colour lying between $\sim 1.5$ and 5 . They use an extrapolation to the bluer colour for many $\mathrm{C}$ stars. Our stars are $\mathrm{M}$ type, and almost none are as red as that. We adopted another approach based scaling the observed $V$-band amplitude $\Delta V$. We compare this quantity with the difference between $K_{s}$ from 2MASS and $K_{s}$ from the DENIS survey (Epchtein et al. 1999). These surveys provide independent data taken at two different dates. DENIS and 2MASS have very similar $K$-band photometries: according to Carpenter (2001), $K_{s}$ (DENIS) is smaller than $K_{s}$ (2MASS), by $\sim 0.02 \mathrm{mag}$ for the range of $\left(J-K_{S}\right)_{0}$ of our stars. Therefore, we expect that, for a given $\Delta V$, the $K_{s}$ difference can reach the $K_{s}$ amplitude (all along this work, amplitudes mean peak-to-peak amplitudes).

By cross-matching our sample with the DENIS catalogue and requesting $K_{s}>5$ to avoid saturation results in 136 stars. Figure 8 shows this approach to work reasonably well. The average shift between the two photometries was found to be 0.05 mag. In Fig. 8, the error bars drawn in ordinates are derived from summing in quadrature the 2MASS and DENIS catalogue errors. On abcissa, the following errors, which we believe

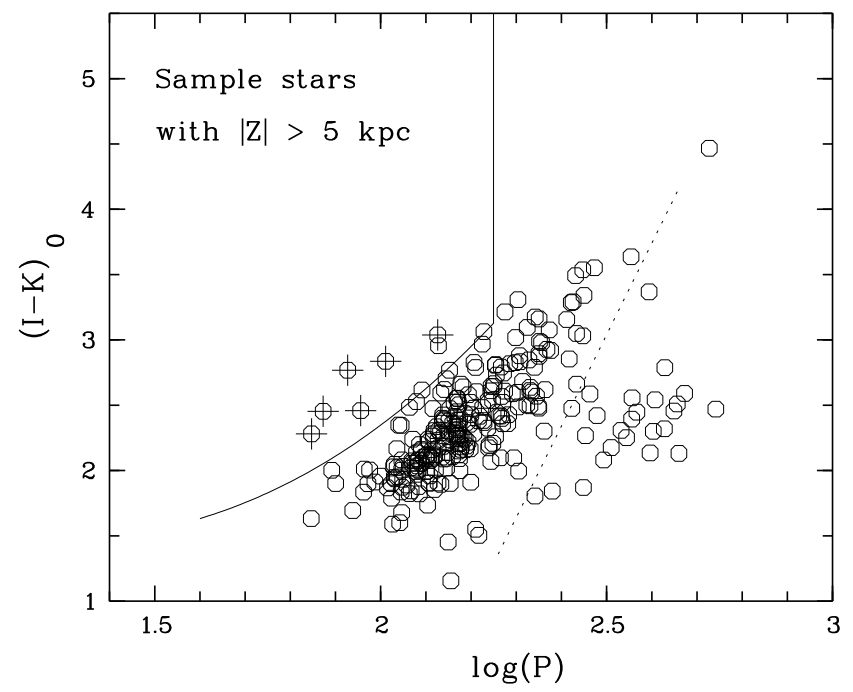

Fig. 6. $\left(I-K_{s}\right)_{0}$ versus period (in days) for our sample stars located at more than $5 \mathrm{kpc}$ from the Galactic plane. The curved line is the same as for LMC. Stars with an overplotted cross are attributed to sequence $C^{\prime}$. Stars to the right of dotted line are considered to belong to sequence D.

reasonable, were adopted: \pm 0.15 mag when $\Delta V=2.5$ mag down to \pm 0.05 mag if $\Delta V=0.3$. This plot shows that $\Delta K_{s} \approx 0.40 \times \Delta V$. For example, when $\Delta V=2 \mathrm{mag}$, Fig. 8 shows that the absolute value between the two $K_{s}$ values can reach $0.8 \mathrm{mag}$.

After including the $\sim 0.2$ mag error discussed in Sect. 3.1, the last uncertainty term (called d above) can reach $0.5 \times(0.4(\Delta V+$ $0.2)$ ) mag. Typically, $\Delta K_{s} \sim 0.1 \mathrm{mag}$ for $\Delta V=0.3$, and $\Delta K_{s} \sim$ $0.4 \mathrm{mag}$ for $\Delta V=2$. The two last uncertainties (named $\mathrm{c}$ and d above) dominate, and distances are derived to within $20 \%$ or better.

The derived distance can be large, up to $150 \mathrm{kpc}$. In Fig. 9, we plot the distances as a function of the longitude $\tilde{\Lambda}_{\odot}$ in the plane of the Sgr orbit. Coordinates in this plane $\tilde{\Lambda}_{\odot}$ and $\widetilde{B}_{\odot}$ are derived from Appendix A of Belokurov et al. (2014). Here, we have considered objects that have $\left|\tilde{B}_{\odot}\right|<13^{\circ}$. The continuous line traces the mean locus of RR Lyr stars as given by Hernitschek et al. (2017). The $1 \sigma$ depth of the RR Lyrae location is about $4 \mathrm{kpc}$ and $6 \mathrm{kpc}$ for the leading and trailing arm, respectively. For the leading arm, there is reasonable agreement between RR Lyrae and $\mathrm{M}$ stars. For example, near $\tilde{\Lambda}_{\odot}=50^{\circ}$ and $90^{\circ}$, agreement is very good. However, for $\tilde{\Lambda}_{\odot}=60-70^{\circ}$, many of our M stars are slightly farther away from us. Concerning the trailing arm, the 


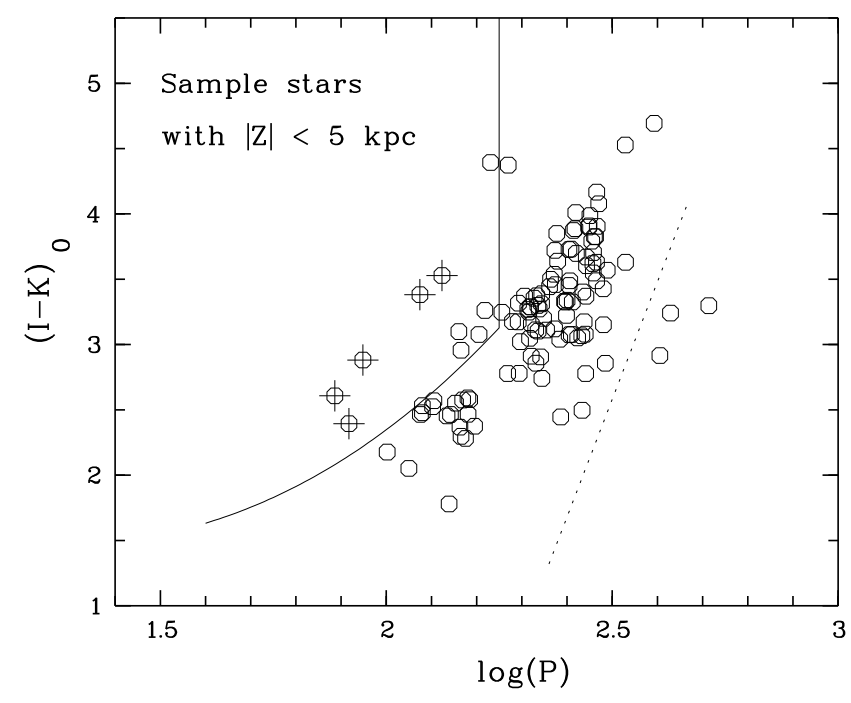

Fig. 7. Same as Fig. 6 for stars of our sample that are closer than $5 \mathrm{kpc}$ from the Galactic plane.

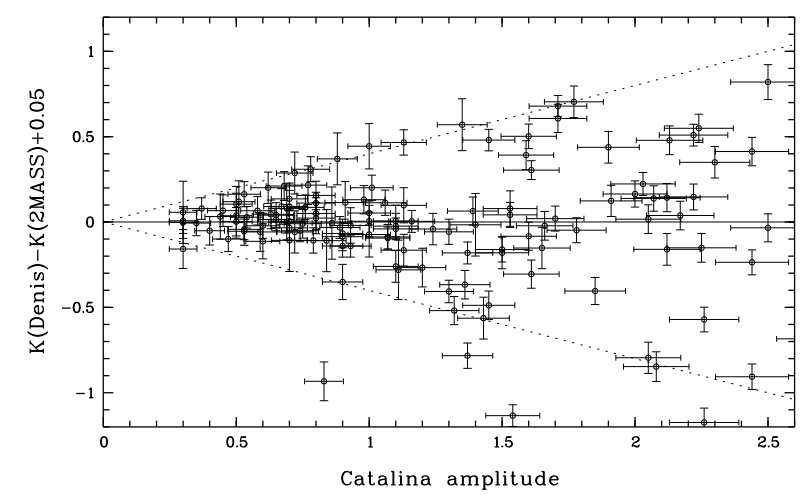

Fig. 8. Difference between $K_{\text {DENIS }}$ and $K_{2 \text { MASS }}$ as a function of the Catalina amplitude for 136 stars of our sample obeying $K_{2 \text { MASS }}>5$. This diagram suggests the $K$-band amplitude to be around $0.4 \times$ the $V$ band Catalina amplitude, as indicated by dotted lines.

distances are in agreement for $70 \%$ of the stars, but are larger (by up to $40 \%$ ) for $30 \%$ of them. MMK-101 is at a distance suggesting it is member of a Sgr arm extension called "feature 2" in Sesar et al. (2017).

At $\tilde{\Lambda}_{\odot} \sim 120^{\circ}$, we indicate two stars, MMK-153 and MMK-154, at distances $92 \pm 18 \mathrm{kpc}$ and $98 \pm 13 \mathrm{kpc}$, respectively. Coordinates and some photometric information of stars discussed in the text are provided in Table 2. MMK-153 and MMK-154 belong to the globular cluster Pal 4 and were already known. According to Harris (1996, 2010 edition), this cluster is at $108 \mathrm{kpc}$, with a metallicity $\mathrm{Fe} / \mathrm{H}=-1.41$. At distances of $100 \mathrm{kpc}$, they represent excellent examples on which other stars can be compared. In particular, obtaining the Pal 4 distance within uncertainties for these two stars supports our method for distance determinations.

In the upper right corner of Fig. 9, we have labelled MMK003 and MMK-007, because they are among the most distant stars in our sample. They are angularly separated by only $5.7^{\circ}$. Interestingly, they are also close to two carbon stars, renamed HG3 and HG5 by HG2015. The former is at $2.6^{\circ}$ from MMK003 , and the latter is at $6.5^{\circ}$ from MMK-007. HG2015 attributed HG3 to the A16 halo sub-structure, while this was less sure for HG5. Given uncertainties, the distances of our M stars, $145 \pm 20 \mathrm{kpc}$, are in remarkable agreement with those of the
Table 2. Coordinates (J2000) from 2MASS, de-redenned 2MASS $K_{s}$ magnitudes, and periods (from Catalina) for MMK stars cited in the text.

\begin{tabular}{lrrrr}
\hline \hline Star & RA $($ deg $)$ & Dec $($ deg $)$ & $K_{0}$ & $P($ days $)$ \\
\hline MMK-003 & 8.55187 & -1.53182 & 14.15 & 142 \\
MMK-007 & 13.15590 & -3.61278 & 13.85 & 160 \\
MMK-092 & 108.00498 & 32.71524 & 13.39 & 127 \\
MMK-094 & 110.00952 & 32.70439 & 13.32 & 109 \\
MMK-095 & 110.47729 & 33.50544 & 12.66 & 224 \\
MMK-101 & 120.07031 & 32.16365 & 13.80 & 134 \\
MMK-114 & 132.35449 & 20.98200 & 13.82 & 297 \\
MMK-153 & 172.30163 & 28.97090 & 13.06 & 150 \\
MMK-154 & 172.31129 & 28.97068 & 13.59 & 130 \\
\hline
\end{tabular}

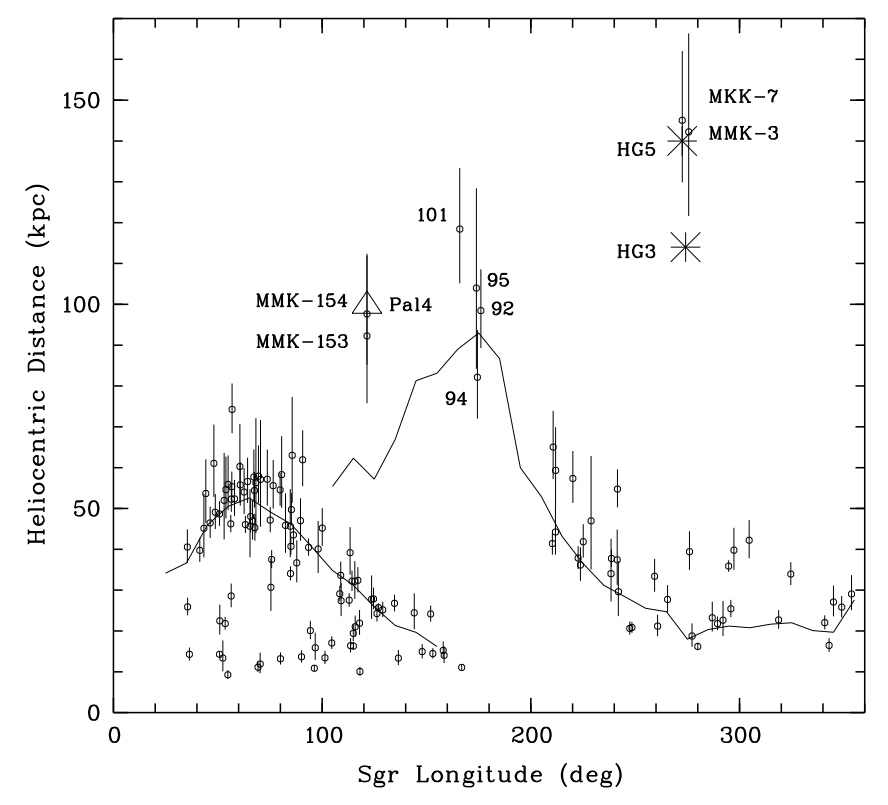

Fig. 9. Heliocentric distance as a function of the Sgr longitude. Only stars within $13^{\circ}$ of the Sgr orbit plane are plotted. The continuous line is the average position of RR Lyr variables of the Sgr arms given by Hernitschek et al. (2017). The $1 \sigma$ scatter of this RR Lyrae position is $\sim 5 \mathrm{kpc}$. The globular cluster Pal 4 is indicated by a triangle. Two carbon stars, HG5 and HG3, are plotted with asterisks.

carbon stars, $114 \pm 15$ and $140 \pm 22 \mathrm{kpc}$. The light curves of the eight more distant stars shown in Fig. 9 are given in Fig. 10. Periods are those given by the Catalina DR2 database. The two light curves plotted at bottom of Fig. 10 are MMK-153 and MMK154, members of Pal 4.

\subsection{A peculiar clump}

When one looks at Fig. 4, middle panel, one can see that, south of this line, there exists a group of stars at RA $\sim 160^{\circ}$. This part of the sky is magnified in Fig. 11, where we plot $\mathrm{M}$ stars (circles), known C stars (filled circles), together with the two stars of pair 6 of Starkenburg et al. (2009). Crosses indicate those objects at more than $25 \mathrm{kpc}$. The three $\mathrm{C}$ stars are, from bottom to top, m7 (alias HG52), m36 (alias HG 48), and m04 (Mauron et al. 2004). M04 was missed in the census of HG2015. The distances and heliocentric radial velocities $V_{\mathrm{r}}$ of these three $\mathrm{C}$ stars are $40 \mathrm{kpc}$ and $+342 \mathrm{~km} \mathrm{~s}^{-1}, 24 \mathrm{kpc}$ and $+306 \mathrm{~km} \mathrm{~s}^{-1}$, and $75 \mathrm{kpc}$ and $+202 \mathrm{~km} \mathrm{~s}^{-1}$, respectively. As noted by HG2015, the first of 

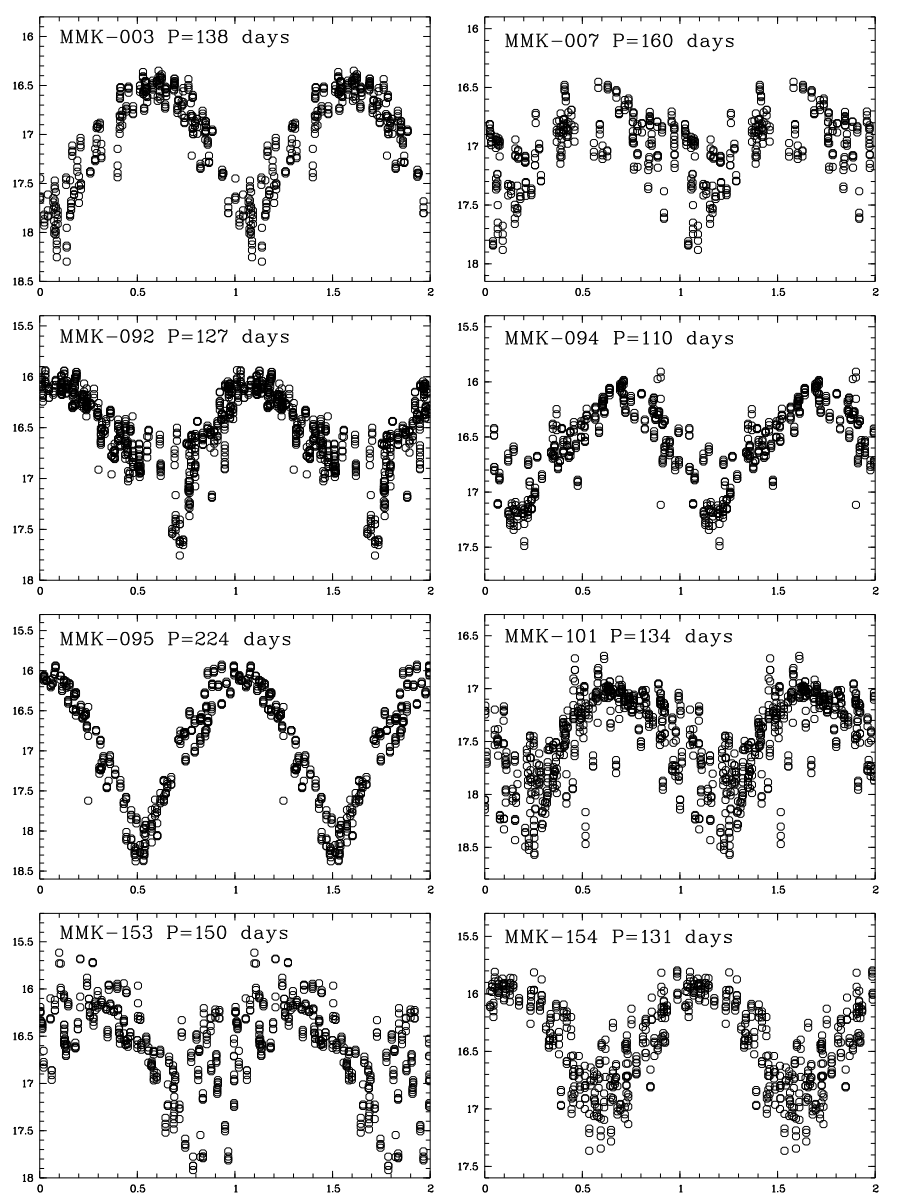

Fig. 10. Catalina magnitude as a function of phase, for labelled stars in Fig. 9. The light curves at bottom refer to two members of the Pal 4 globular cluster.

these three stars may have a link with the Starkenburg et al. stars located at $30 \mathrm{kpc}$ with $V_{\mathrm{r}}=380 \mathrm{~km} \mathrm{~s}^{-1}$. The other two C stars are isolated. We have also indicated the position of the Orphan stream which is well detected by a variety of methods (Grillmair 2006; Belokurov et al. 2006; Bernard et al. 2016) and is at a distance of $\sim 20 \mathrm{kpc}$. It is clear that our clump is not the Orphan stream. Radial velocities are necessary to confirm or deny the physical reality of this peculiar feature.

\subsection{Gaia data}

\subsubsection{Parallaxes}

In the Gaia DR2 (Gaia collaboration 2016), parallaxes are available for all the sample stars but two. However, half of them are negative and the positive ones have large uncertainties. In the whole sample, only seven stars have $\mid \epsilon(\pi) / \pi) \mid<0.2$. Therefore, it is necessary to wait for more robust Gaia measurements and future data releases in order to compare them to our distances based on period-luminosity relations.

\subsubsection{Radial velocities}

We first collected all Gaia radial velocities. We added those from the APOGEE (Majewski et al. 2017) and RAVE (Steinmetz et al. 2006; Kordopatis et al. 2013) surveys, and a few from Monaco et al. (2007). In most cases there is excellent agreement between them when two measurements are available, with differences of $\leq 2 \mathrm{~km} \mathrm{~s}^{-1}$. Our catalogue includes these velocities, their errors and references. Some additional velocities come from Feast \& Whitelock (2000), and from Smak \& Preston (1965), with errors $\sim 5 \mathrm{~km} \mathrm{~s}^{-1}$. Although less accurate, $\sim 12-15 \mathrm{~km} \mathrm{~s}^{-1}$, a few velocities come from the LAMOST database (Luo et al. 2016) or the Sloan DR7 M dwarf catalogue (West et al. 2011). Overall, of 429 stars on our catalogue, 90 have a radial velocity.

\subsubsection{Proper motions}

Gaia DR2 proper motions allow us to discard some distant stars, because these proper motions would imply an excessive tangential velocity $\left(V_{\mathrm{t}}\right)$ (we attributed a negative flag in our catalogue; see below). The relevant relation is $V_{\mathrm{t}}=4.7 \mu \times D \mathrm{~km} \mathrm{~s}^{-1}$

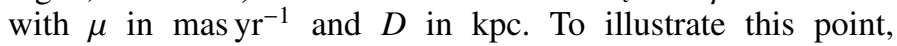
two of the most distant stars in the Galaxy were found by Bochanski et al. (2014), namely ULAS J001535.72+015549.6 and ULAS J074417.48+25253233.0, at proposed distances of $\sim 275 \mathrm{kpc}$, and $\sim 240 \mathrm{kpc}$, with uncertainties of $\sim 70 \mathrm{kpc}$. These distances are based on the result that stars would be M-giants, and not dwarfs. Both are interestingly close to the Sgr mean orbit plane: $\tilde{B}_{\odot}=+16.3^{\circ}$ and $\tilde{B}_{\odot}=-4.7^{\circ}$, for the first and second stars, respectively. While the second object has no significant Gaia proper motion, the first displays $\mu_{\alpha} \cos (\delta)=$ $-5.00 \pm 1.11 \mathrm{mas} \mathrm{yr}^{-1}$ and $\mu_{\delta}=-4.91 \pm 0.82 \mathrm{mas} \mathrm{yr}^{-1}$. Were its distance of $240 \mathrm{kpc}$ correct, it would yield a tangential velocity of $\sim 9000 \mathrm{~km} \mathrm{~s}^{-1}$. It seems to us more probable that it is a much closer dwarf star, although considerable care was dedicated by Bochanski et al. (2014) to spectroscopically discriminating giants and dwarfs.

Another very distant object was proposed by Mauron et al. (2018), named MMK-114 here, with $K_{s}=13.8$ and $\left(J-K_{s}\right)_{0}=$ $1.39, B_{\text {Sgr }}=-9.2^{\circ}$. The colour makes it unclear to classify it $\mathrm{M}$ or C. Its light curve (Fig. 12) is compatible with a semi-regular variable having a peak-to-peak amplitude of 0.6 mag. Catalina DR2 provides a tentative period of 297 days. Because it is faint, adopting this period would yield a distance of $\sim 200 \mathrm{kpc}$, putting it outside of the frame of Fig. 9. Gaia DR2 does not provide a significant parallax but gives $\mu_{\alpha} \cos (\delta)=-0.22 \pm 0.26$ mas yr$^{-1}$ and $\mu_{\delta}=-2.1 \pm 0.19{\text { mas } \mathrm{yr}^{-1}}^{-1}$. Provided this $\delta$ motion is confirmed, this star is closer than $200 \mathrm{kpc}$ in order, again, to avoid excessively large velocity $\left(\sim 2000 \mathrm{~km} \mathrm{~s}^{-1}\right)$.

The stars MMK-003, MMK-007, MMK-101, MMK-153, MMK-154 have no significant proper motion. Those close to the apocentre of the trailing arm, MMK-92, MMK-094, MMK-101 have plausible $V_{\mathrm{t}}=400,240$, and $270 \mathrm{~km} \mathrm{~s}^{-1}$ at their respective distances. We also checked the eight carbon stars listed by Deason et al. (2012, their Table 2), and found that only J14460055 displays some motion at more than $3 \sigma: \mu_{\alpha} \cos (\delta)=-1.00 \pm$ $0.12 \mathrm{mas} \mathrm{yr}^{-1}$ and $\mu_{\delta}=-0.53 \pm 0.10$ mas $^{-1}$. At its distance of $\sim 80 \mathrm{kpc}$, this yields a plausible $V_{\mathrm{t}}=425 \mathrm{~km} \mathrm{~s}^{-1}$.

\subsection{The catalogue}

Our catalogue includes the MMK number, $\alpha, \delta$ (J2000), $l$ and $b$, Sgr orbit coordinates $\tilde{\Lambda}_{\odot}$ and $\tilde{B}_{\odot}, E(B-V),\left(K_{s}\right)_{0},(J-$ $\left.K_{s}\right)_{0}$, absolute magnitude $M_{K_{s}}$, distance and its error in kpc, $Z$ in kpc, period in days, Catalina amplitude and time-averaged magnitude $V_{\mathrm{CSS}}$, radial velocity, its uncertainty and the corresponding source, the $V_{\mathrm{r}}$ source, a quality and a corresponding comment, and finally the 2MASS and CRTS names when available. The first three lines of the catalogue are given in Table 3 in an abridged version for clarity. 


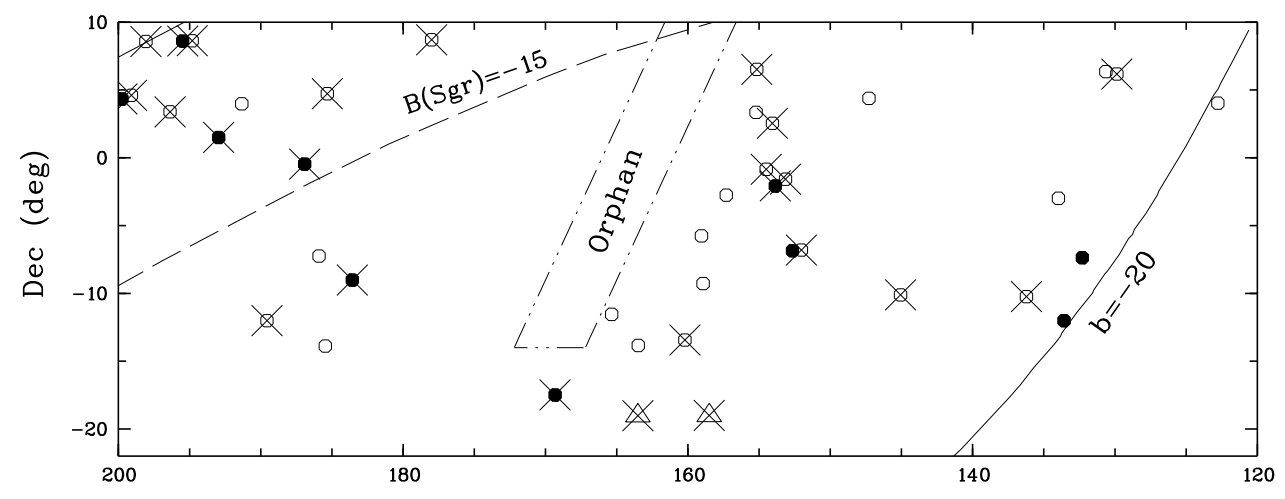

R.A. (deg)
Fig. 11. Close-up on the region of the group of stars seen at $\mathrm{RA} \sim 160^{\circ}$. M stars and $\mathrm{C}$ stars are plotted with open and filled circles, respectively. Two triangles at bottom are pair 6 of Starkenburg et al. (2009). Overplotted crosses are stars more distant than $25 \mathrm{kpc}$ from the Sun. Those at the upper left corner belong to Sgr arm. The Orphan stream position is indicated.

Table 3. Abridged version of the catalogue of $M$ halo northern long period variables.

\begin{tabular}{lrrrrrrrrrrrrr}
\hline \hline Star & $\alpha$ & $\delta$ & $E_{B-V}$ & $\left(K_{s}\right)_{0}$ & $\left(J-K_{s}\right)_{0}$ & $M_{K S}$ & $D$ & $\delta D$ & $P$ & $\Delta V$ & $V_{\mathrm{CSS}}$ & $V_{\mathrm{r}}$ & $\delta V_{\mathrm{r}}$ \\
\hline MMK-001 & 4.06675 & -17.60342 & 0.039 & 10.39 & 1.10 & -6.44 & 23.2 & 4.0 & 134.0 & 1.5 & 13.83 & -59.42 & 6.8 \\
MMK-002 & 5.64850 & -5.20222 & 0.026 & 9.81 & 1.07 & -6.24 & 16.2 & 1.5 & 424.4 & 0.4 & 13.49 & -116.00 & 0.3 \\
MMK-003 & 8.55188 & -1.53182 & 0.019 & 14.15 & 0.93 & -6.62 & 142.2 & 24.5 & 138.2 & 1.5 & 17.01 & -9999.00 & 0.0 \\
\hline
\end{tabular}

Notes. The full table is available at the CDS.

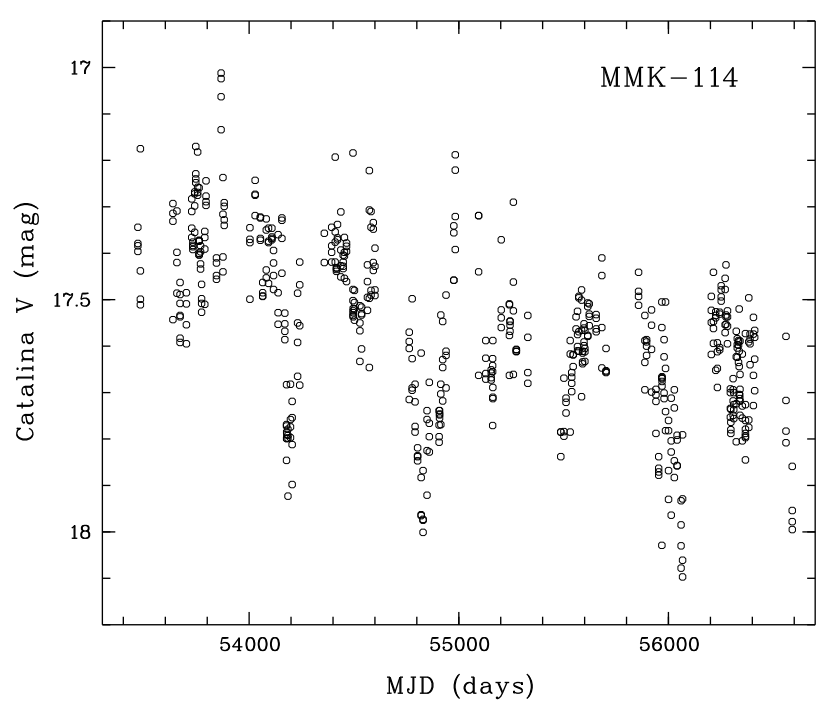

Fig. 12. Light curve of MMK-114 (see text).

\section{Conclusions}

This work can be summarised as follows:

(1) By considering available literature on long-period variables out of the Galactic plane and at $\delta>-20^{\circ}$, our goal was to build a catalogue of AGB M stars located in the halo. This catalogue will make a northern complement to the census of $\mathrm{C}$ stars established by Huxor \& Grebel (2015). After merging and cleaning available catalogues, a sample of 417 pulsating stars in the northern hemisphere is achieved, which includes no known $\mathrm{C}$ stars. Of these 417 stars, 262 are located at more than $\sim 5 \mathrm{kpc}$ from the Galactic plane. Consideration of their $\left(J-K_{s}\right)_{0}$ colour, periods, and amplitudes suggests that they are $M$ stars.

(2) By selecting stars far away from the Galactic plane $(|Z|>$ $5 \mathrm{kpc}$ ), one confirms a strong concentration along the apparent, orbital path of the Sgr tidal arms, especially on the leading arm.
A major finding of this study is that there are about three times more $\mathrm{M}$ stars than $\mathrm{C}$ stars. This offers a future opportunity to investigate the Sgr AGB population in more detail and analyse it in a complementary way to $\mathrm{C}$ stars.

(3) The pulsation modes, needed for distance determination, are found with a diagram showing the $\left(I-K_{s}\right)_{0}$ colour as a function of period, and by comparing it to that of the Large Magellanic Cloud. As for C stars, very few M stars are found to be on sequence $C^{\prime}$. Then, near-infrared Wesenheit P-L relations established for LMC oxygen-rich AGB stars are adopted. Distances up to $\sim 150 \mathrm{kpc}$ are derived, with typical relative uncertainties better than $20 \%$. Two pulsating stars found in the Pal 4 globular cluster have correct distances of $100 \mathrm{kpc}$.

(4) If one considers stars located within $13^{\circ}$ from the Sgr mean orbit plane, our distances for the leading arm agree very well with those recently derived with RR Lyr stars. Some of them are somewhat larger in the trailing arm. We find four stars located at the apocentre. Two stars are found to be close to two carbon stars in the A16 sub-structure at $\sim 150 \mathrm{kpc}$. There are seven stars angulary close to the Sgr orbit plane and more distant than $70 \mathrm{kpc}$. We also draw attention to a clump of $\mathrm{M}$ and $\mathrm{C}$ stars in the halo angularly close to the Orphan stream, but distinct of it. Radial velocities are required to check conclusions more rigorously in these cases.

(5) A catalogue of M AGB stars is presented. These stars belong to the thick disk, the halo, and the Sgr arms. Ninety radial velocities were collected from Gaia and other surveys like RAVE. Significant proper motions were used to check supposedly large distances, but few are discarded and flagged in the catalogue. Gaia DR2 parallaxes are presently too uncertain to achieve a robust comparison with our distances. The catalogue (Table 3) is available on Vizier at CDS, or on request. Further investigation of our sample with additional radial velocities and Gaia variability information will be presented in a future paper.

Acknowledgements. N.M. thanks our anonymous referee for remarks that greatly improved the manuscript. We also thank Olivier Richard for generous 
help, Henri Reboul and Denis Puy for encouragments, and the staff of LUPM for kind assistance. We thank Michael Feast, Patrick de Laverny and Nina Hernitschek for comments. N.M. is grateful to the Université de Montpellier for support. This work makes use of the Catalina database (California Institute of Technology, NASA), the Lincoln Near-Earth Asteroid Research LINEAR database (Massachusetts Institute of Technology Lincoln Laboratory, NASA and US Air Force), the Two Micron All Sky Survey (University of Massachusetts and IPAC/Caltech funded by NASA and NSF), the European infrared photometry DENIS experiment. We exploited the data of the European Space Agency mission Gaia processed by the Gaia Data Processing and Analysis Consortium, and the SIMBAD/Vizier facilities, offered by and operated at CDS, Strasbourg, France.

\section{References}

Alcock, C., Allsman, R. A., Alves, D., et al. 1997, ApJ, 486, 607 Belokurov, V., Zucker, B. D., Evans, N. W., et al. 2006, MNRAS, 642, L137

Belokurov, V., Koposov, S. E., Evans, N. W., et al. 2014, MNRAS, 437, 116

Bernard, E. J., Ferguson, A. M. N., Schlafly, E. F., et al. 2016, MNRAS, 463, 1759

Bochanski, J. J., Willman, B. W., Caldwell, N., et al. 2014, ApJ, 790, L5

Cardelli, J. A., Clayton, G. C., \& Mathis, J. S. 1989, ApJ, 345, 245

Carlin, J. L., Sheffield, A. A., Cunha, K., \& Smith, V. V. 2018, ApJ, 859, L10

Carpenter, J. M. 2001, AJ, 121, 2851

Chou, M.-Y., Majewski, S. R., Cunha, K., et al. 2007, ApJ, 670, 346

Deason, A. J., Belokurov, V., Evans, N. W., et al. 2012, MNRAS, 425, 2840

De Grijs, R., Wicker, J. E., \& Bono, G. 2014, AJ, 147, 122

Drake, A. J. 2006, AJ, 131, 1044

Drake, A. J., Djorgovski, S. G., Mahabal, A., et al. 2009, ApJ, 696, 870

Drake, A. J., Graham, M. J., Djorgovski, S. G., et al. 2014, ApJS, 213, 9

Epchtein, N., Deule, E., Derrière, S., et al. 1999, A\&A, 349, 236

Feast, M., \& Whitelock, P. 2000, MNRAS, 317, 460

Gaia collaboration (Prusti, T., et al.) 2016, A\&A, 595, A1

Gigoyan, K. S., \& Mickaelian, A. M. 2012, MNRAS, 419, 3346

Grillmair, C. J. 2006, ApJ, 645, L37

Habing, H. J., \& Olofsson, H. 2004, Asymptotic Giant Branch Stars (New York Springer)

Harris, W. E. 1996, AJ, 112, 1487

Hernitschek, N., Sesar, B., Rix, H.-W., et al. 2017, ApJ, 850, 96

Huxor, A. P., \& Grebel, E. K. 2015, MNRAS, 453, 2653

Ita, Y., Tanabé, T., Matsunaga, N., et al. 2004, MNRAS, 347, 720

Jura, M., \& Kleinman, S. G. 1992, ApJS, 79, 105

Kordopatis, G., Gilmore, G., Steinmetz, M., et al. 2013, AJ, 146, 134

Law, D. R., \& Majewski, S. R. 2010, ApJ, 714, 229

Luo, A. L., Zhao, Y. H., Zhao, G., et al. 2016, VizieR Online Data Catalog: V/149

Majewski, S. R., Schiavon, R. P., Frinchaboy, P. M., et al. 2017, AJ, 154, 94

Mauron, N., Azzopardi, M., Gigoyan, K., \& Kendall, T. R. 2004, A\&A, 418, 77

Mauron, N., Gigoyan, K., \& Kostandyan, G. R. 2018, Astrophysics, 61, 10
Monaco, L., Bellazzini, M., Bonifacio, P., et al. 2007, A\&A, 464, 201 Palaversa, L., Ivezić, Z., Eyer, L., et al. 2013, AJ, 146, 10

Samus, N. N., Kazarovets, E. V., Durlevitch, O. V., et al. 2017, Astron. Rep., 61, 80

Schlegel, D. J., Finkbeiner, D. P., \& Davis, M. 1998, ApJ, 500, 525

Schneider, D. P., Richards, G. T., Hall, P. B., et al. 2010, AJ, 139, 2360

Sesar, B., Hernitschek, N., Dierickx, M. I. P., et al. 2017, ApJ, 844, L4

Smak, J. I., \& Preston, G. W. 1965, ApJ, 142, 943

Soszyński, I., Dziembowski, W. A., Udalski, A., et al. 2007, Acta Astron., 57, 201

Starkenburg, E., Helmi, A., Morrison, H. L., et al. 2009, ApJ, 698, 597

Steinmetz, M., Zwitter, T., Siebert, A., et al. 2006, AJ, 132, 1645

Udalski, A., Szymanski, M., Stanek, K., et al. 1994, Acta Astron., 44, 165

van Loon, J Th, van Leeuwen, F., Smalley, B., et al. 2007, MNRAS, 382, 1353

Véron-Cetty, M.-P., \& Véron, P. 2010, A\&A, 518, A10

West, A. A., Morgan, D. P., Bochanski, J., et al. 2011, AJ, 141, 97

Whitelock, P., Menzies, J., Feast, M., et al. 1994, MNRAS, 267, 711

Whitelock, P., Menzies, J., Feast, M., et al. 1995, MNRAS, 276, 219

Wood, P. R., Alcock, C., Allsman, R. A., et al. 1999, IAU Symp., 191, 151

\section{Appendix A: Estimation of the l-band photometry}

The goal of this appendix is to describe how we estimated the $I_{0}$-band time-averaged photometry of our sample, which is needed to separate sequence $\mathrm{C}$ and $\mathrm{C}^{\prime}$. From our sample of 417 stars, we identified a sub-sample of 96 stars with good quality $I$-band photometry from the DENIS catalogue (uncertainty on $I<0.2 \mathrm{mag}$, $90 \%$ of them with $<0.05 \mathrm{mag}$ error). Then, the Catalina $V_{\mathrm{CSS}}$, the 2MASS $J \& K_{s}$, and the DENIS $I$ are corrected for interstellar extinction by adopting the colour excess from Schlegel et al. (1998). The used extinction law is from Cardelli et al. (1989), that is, $A_{V}=3.107 E(B-V), A_{I}=1.705 E(B-V), A_{J}=0.804 E(B-$ $V), A_{K}=0.342 E(B-V)$. Finally, we searched for a function $f\left(\left(V_{\mathrm{CSS}}\right)_{0}, J_{0},\left(K_{S}\right)_{0}\right)$ that is as close as possible to the observed $I_{0}$. We find that the best fit relation is:

$I_{0}=J_{0}-0.22+0.58\left(\left(V_{\mathrm{CSS}}\right)_{0}-J_{0}\right)+0.05\left(\left(V_{\mathrm{CSS}}\right)_{0}-13.5\right)^{2}$

This fit is found with $N=96$ stars, with a scatter $\sigma=$ 0.22 mag. Outliers at more than $2.2 \sigma$ were removed. We checked that the residuals do not depend on color such as $\left(V_{\mathrm{CSS}}-\left(K_{S}\right)_{0}\right)$. These 96 stars have the following properties with $10.5<$ $\left(V_{\mathrm{CSS}}\right)_{0}<17,2.6<\left(V-K_{S}\right)_{0}<5.6,0.75<\left(J-K_{S}\right)<$ $1.4, E(B-V)<0.3$. These properties are obeyed by $80 \%$ of stars in our sample. 